\title{
Contagem, isolamento e caracterização de bactérias psicrotróficas contaminantes de leite cru refrigerado
}

\author{
Counting, isolation and characterization of psychrotrophic bacteria from refrigerated raw milk \\ ${\text { Edna Froeder Arcuri }{ }^{*} \text { Priscilla Diniz Lima da Silva }{ }^{\mathrm{II}} \text { Maria Aparecida Vasconcelos Paiva Brito }}^{\mathrm{I}}$
José Renaldi Feitosa Brito $^{\mathrm{I}}$ Carla Christine Lange ${ }^{\mathrm{I}}$ Margarida Maria dos Anjos Magalhães $^{\mathrm{II}}$
}

RESUMO

Com os objetivos de quantificar, isolar $e$ caracterizar bactérias psicrotróficas contaminantes de leite cru refrigerado, produzido na região da Zona da Mata de Minas Gerais e Sudeste do Rio de Janeiro, foram analisadas amostras de leite coletadas de 20 tanques coletivos e 23 tanques individuais. As contagens de bactérias psicrotróficas nas amostras de leite para os dois tipos de tanques de refrigeração variaram entre $10^{2}$ e $10^{7}$ Unidades Formadoras de Colônias (UFC) $\mathrm{ml}^{-1}$, porém, um maior número de tanques coletivos

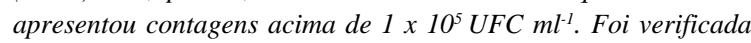
a predominância de bactérias psicrotróficas gram-negativas (81,2\%), que foram identificadas pelos sistemas API $20 \mathrm{E} e$ API 20NE nos gêneros: Aeromonas, Alcaligenes, Acinetobacter, Burkholderia,Chryseomonas, Enterobacter, Ewingella, Klebsiella, Hafnia, Methylobacterium, Moraxella, Pantoea, Pseudomonas, Serratia, Sphingomonas e Yersinia. As bactérias gram-positivas (18,8\%) foram identificadas com API $50 \mathrm{CH}$, API Coryne e API Staph, nos gêneros: Bacillus, Brevibacterium, Cellum/Microbacterium, Kurthia $e$ Staphylococcus. Os sistemas API utilizados não identificaram todos os isolados bacterianos. Pseudomonas foi o gênero mais isolado e P. fluorescens foi a espécie predominante. A maioria dos isolados bacterianos apresentou atividade proteolítica e/ ou lipolítica a temperaturas de refrigeração de $4^{\circ} \mathrm{C}, 7^{\circ} \mathrm{C} e$ $10^{\circ} \mathrm{C}$, evidenciando seu alto potencial de deterioração do leite e dos produtos lácteos. Os resultados ressaltam que maior atenção deve ser dada aos procedimentos que impeçam a contaminação do leite por esses microrganismos.

Palavras-chave: bactérias psicrotróficas gram-negativas, bactérias psicrotróficas gram-positivas, proteases, lípases.

\section{ABSTRACT}

This study aimed to quantify, isolate and characterize psychrotrophic bacteria from refrigerated raw milk produced at the 'Mata' Region of Minas Gerais State and Southeast of Rio de Janeiro State, Brazil. Raw milk samples, were collected at the farms, from 20 collective refrigerated tanks and 23 individual refrigerated tanks. The psychrotrophic bacteria counting ranged from $10^{2}$ to $10^{7}$ Colony Forming Units (CFU) $\mathrm{ml}^{-1}$ for both types of refrigerated tanks, but most of the collective tanks showed counts higher than $1 \times 10^{5} \mathrm{CFU}$ $\mathrm{ml}^{-1}$. Predominance of psychrotrophic gram-negative bacteria (81.2\%), that were identified by API 20E and API 20NE as belonging to genera: Aeromonas, Alcaligenes, Acinetobacter, Burkholderia, Chryseomonas, Enterobacter, Ewingella, Klebsiella, Hafnia, Methylobacterium, Moraxella, Pantoea, Pseudomonas, Serratia, Sphingomonas e Yersinia were oserved. The gram-positive bacteria (18.8\%), were identified by API 50 $\mathrm{CH}$, API Coryne and API Staph, to genera: Bacillus, Brevibacterium, Cellum / Microbacterium, Kurthia $e$ Staphylococcus. The API systems utilized could not identify all the bacterial isolates. Pseudomonas was the genus most isolated with P. fluorescens as the predominant species. Most of the isolates presented proteolytic and/or lipolytic activity at $4^{\circ} \mathrm{C}$, $7^{\circ} \mathrm{C}$ and $10^{\circ} \mathrm{C}$ showing high potential for milk and milk products spoilage. The results indicated that more attention must be taken to the procedures necessaries to reduce milk contamination with psychrotrophic bacteria.
Key words: Psychrotrophic gram-negative bacteria, Psychrotrophic gram-positive bacteria proteases, lipases.

'Embrapa Gado de Leite. Rua Eugênio do Nascimento, 610, Bairro Dom Bosco, 36038-330, Juiz de Fora, MG, Brasil. E-mail: edna@cnpgl.embrapa.br. *Autor para correspondência.

${ }^{I}$ Departamento de Engenharia Química, Universidade Federal do Rio Grande do Norte (UFRN), 59072-970, Campus Universitário, Natal, RN, Brasil. 


\section{INTRODUÇÃO}

Bactérias psicrotróficas são aquelas capazes de se desenvolver em temperaturas abaixo de $7^{\circ} \mathrm{C}$ (FRANK et al., 1992), sendo os principais agentes de deterioração de leite cru refrigerado e de seus derivados. A ação deterioradora das bactérias psicrotróficas se deve principalmente à produção de proteases, lípases e fosfolipases, que hidrolisam respectivamente a proteína e a gordura do leite. A maioria das bactérias psicrotróficas não sobrevive à pasteurização, porém, muitas de suas enzimas hidrolíticas são termorresistentes, podendo resistir mesmo ao tratamento UHT e permanecerem ativas. A presença de enzimas termoestáveis no leite cru é especialmente prejudicial para a qualidade do leite UHT devido à sua estocagem à temperatura ambiente por longos períodos de tempo. Outros defeitos dessas enzimas incluem alterações de sabor e odor em diversos produtos e redução do rendimento dos queijos (CHAMPAGNE et al., 1994; SØRHOUG \& STEPANIAK, 1997; CHEN et al., 2003).

O grupo de microrganismos psicrotróficos inclui bactérias gram-negativas e gram-positivas. Os principais gêneros isolados, em estudos conduzidos em países de clima temperado, são: Pseudomonas, Flavobacterium e Alcaligenes (gram-negativas), Clostridium, Microbacterium, Streptococcus, Corynebacterium, Arthrobacter e Bacillus (grampositivas). Bactérias patogênicas como Listeria monocytogenes, Yersinia enterocolitica e algumas estirpes de Bacillus cereus isoladas de leite também são psicrotróficas (SHAH, 1994; SØRHOUG \& STEPANIAK, 1997).

No Brasil, alguns estudos evidenciaram altas contagens de bactérias psicrotróficas em leite cru refrigerado (SOUZA et al., 1999; BRITO et al., 2002; BRUM et al., 2004; PINTO et al., 2006), mas pouco se conhece sobre a composição desta microbiota e suas propriedades hidrolíticas. Os objetivos deste trabalho foram quantificar, isolar e identificar bactérias psicrotróficas contaminantes de leite cru refrigerado na fazenda e avaliar o seu potencial de deterioração.

\section{MATERIAL E MÉTODOS}

Amostras de leite cru refrigerado foram coletadas de 20 tanques coletivos e 23 tanques individuais em propriedades leiteiras, na região da Zona da Mata, Minas Gerais (MG) e na região Sudeste do Rio de Janeiro, durante 15 meses. Após a agitação programada do tanque, foram coletados cerca de $500 \mathrm{~mL}$ de leite utilizando-se um coletor de aço inoxidável e frascos estéreis. As amostras foram transportadas em caixa isotérmica contendo gelo até o Laboratório de Microbiologia da Embrapa Gado de Leite, Juiz de Fora, MG, onde foram analisadas no mesmo dia da coleta.

Para a contagem e o isolamento, diluições das amostras foram plaqueadas em Plate Count Agar (Difco) e as placas foram incubadas a $7^{\circ} \mathrm{C}$ por 10 dias (FRANK et al., 1992). Após a contagem, foram isolada cinco colônias de cada placa em ágar Nutriente (Difco), que foram incubadas a $21^{\circ} \mathrm{C}$ por 24 horas para identificação.

Primeiramente os isolados bacterianos foram avaliados quanto à morfologia celular, à reação de Gram, metabolismo oxidativo e/ou fermentativo da glicose (OF), à produção de oxidase e catalase, ao crescimento em Agar MacConkey (Difco), à motilidade e à presença de esporos (BARROW \& FELTHAN, 1995), para definir o sistema API de identificação (BioMérieux, Marcy l'Etoile, França) a ser usado. A seguir, as bactérias gramnegativas foram identificadas por gênero ou espécie empregando-se os sistemas API 20E e API 20NE e as bactérias gram-positivas empregando-se os sistemas API Staph, API Coryne e API $50 \mathrm{CH}$, de acordo com as orientações do fabricante. Os sistemas API foram repetidos para $50 \%$ dos isolados para garantir a identificação.

A capacidade de produzir proteases foi determinada em ágar caseinato (Merck) e de produzir lípases, em ágar tributirina, segundo FRANK et al. (1992), com incubação a $4^{\circ} \mathrm{C}, 7^{\circ} \mathrm{C}$ e $10^{\circ} \mathrm{C}$ durante 10 dias e a $21^{\circ} \mathrm{C}$ durante três dias. A proteólise e a lipólise são evidenciadas por uma zona clara ao redor da colônia.

\section{RESULTADOS E DISCUSSÃO}

Contagem de bactérias psicrotróficas

O número de bactérias psicrotróficas presentes no leite cru está relacionado às condições higiênicas na produção e ao tempo e à temperatura em que o leite é armazenado. Uma contagem baixa de psicrotróficos no leite é de fundamental importância para sua qualidade, pois a atividade metabólica desses microrganismos resulta em alterações bioquímicas nos constituintes do leite que limitam a vida de prateleira dos produtos lácteos.

As contagens de bactérias psicrotróficas nas amostras de leite para os dois tipos de tanques de refrigeração variaram entre $10^{2}$ e $10^{7}$ Unidades Formadoras de Colônias (UFC) $\mathrm{ml}^{-1}$. A maioria dos tanques individuais apresentou contagens inferiores a $1 \times 10^{5} \mathrm{UFC} \mathrm{ml}^{-1}$ e a maioria dos tanques coletivos apresentou contagens acima deste valor. Esses dados indicam que em muitas propriedades houve deficiência 
nas práticas higiênicas e a mistura de matéria-prima de diversos produtores em tanques coletivos aumentaram os riscos, comprometendo a qualidade final do produto.

Contagens semelhantes de psicrotróficas também foram encontradas por PINTO et al. (2006) para leite de fornecedores de uma indústria de laticínios localizada na Zona da Mata Mineira. Nas 33 amostras de leite coletadas de tanques individuais, as contagens variaram de 2,0 x $10^{2}$ a $1,0 \times 10^{7} \mathrm{UFC} / \mathrm{ml}$, e para 12 tanques coletivos, de $8,9 \times 10^{2}$ a 3,2 $\times 10^{6} \mathrm{UFC} / \mathrm{ml}$.

Os resultados apresentados indicam que há propriedades em que as contagens de bactérias psicrotróficas são muito altas, especialmente em tanques coletivos. Portanto, treinamentos devem ser direcionados para a melhoria das condições de higiene de ordenha e de armazenamento do leite para essas propriedades.

Identificação e caracterização das bacterias psicrotróficas

Das 43 amostras de leite cru refrigerado coletadas nas propriedades rurais, foram isoladas uma levedura e 308 bactérias psicrotróficas, sendo 250 (81,2\%) gram-negativas e 58 (18,8\%) gram-positivas (Tabela 1). A maioria (161) das bactérias gram-negativas foi identificada à espécie: Pseudomonas fluorescens (94), Pseudomonas putida (três), Aeromonas hydrophila (20), Aeromonas sobria (uma), Aeromonas caviae (uma), Burkholderia cepacia (12), Klebsiella oxytoca (10), Ewingella americana (sete), Hafnia alvei (sete),

Tabela 1 - Identificação das bactérias Gram-negativas e Gram-positivas utilizando os sistemas de identificação API e testes bioquímicos.

\begin{tabular}{|c|c|c|}
\hline Gênero/Espécie & Número de estirpes & \% Identificação sistema API \\
\hline \multicolumn{3}{|l|}{ Bactérias Gram-negativas (250) } \\
\hline Pseudomonas fluorescens & 94 & $98,6-99,9$ \\
\hline Pseudomonas putida & 03 & 99,1 \\
\hline Pseudomonas spp. & 11 & $82,3-99,4$ \\
\hline Acinetobacter spp. & 39 & $78,9-91,9$ \\
\hline Aeromonas hydrophila & 20 & $92,9-99.9$ \\
\hline Aeromonas caviae & 1 & 98,9 \\
\hline Aeromonas sobria & 1 & 83,8 \\
\hline Aeromonas spp. & 5 & 85,9-99,9 \\
\hline Pantoea spp. & 17 & $*$ - 99,9 \\
\hline Bukholderia cepacea & 12 & 99,9 \\
\hline Klebsiella oxytoca & 10 & 97,4 \\
\hline Klebsiella sp. & 1 & $*$ \\
\hline Hafnia alvei & 7 & 99,9 \\
\hline Erwingella americana & 7 & $92,9-98,2$ \\
\hline Moraxella spp. & 4 & 82,2 \\
\hline Chryseomonas luteola & 3 & $97,8-99,8$ \\
\hline Serratia spp. & 3 & $*-99,4$ \\
\hline Yersinia spp. & 2 & 95,7 \\
\hline Enterobacter sp. & 1 & $*$ \\
\hline Alcaligenes faecalis & 1 & 96,6 \\
\hline Pasteurella sp. & 1 & 99,3 \\
\hline Sphingomonas paucimobilis & 1 & 99,8 \\
\hline Methylobacterium sp. & 1 & 88,2 \\
\hline Não identificados & 5 & \\
\hline \multicolumn{3}{|l|}{ Bactérias Gram-positivas (58) } \\
\hline Kurthia spp. & 7 & $*$ \\
\hline Bacillus sp. & 1 & $*$ \\
\hline Bacillus coagulans & 1 & 99,2 \\
\hline Bacillus lentus & 2 & 99,1 \\
\hline Brevibacterium sp. & 1 & 96,2 \\
\hline Cellum/Microbacterium & 6 & 99,9 \\
\hline Staphylococcus spp. & 3 & $91,9-97,4$ \\
\hline Não identificada & 37 & \\
\hline
\end{tabular}

*Boa identificação para o gênero, segundo o APILAB Plus v.3.3.3 (bioMérieux).

Ciência Rural, v.38, n.8, nov, 2008. 
Chryseomonas luteola (três), Alcaligenes feacalis (uma), Methylobacterium mesophilicum (uma), Sphingomonas paucimobilis (uma). 84 bactérias foram identificadas a gênero: Pseudomonas (11), Acinetobacter (39), Pantoea (17), Aeromonas (cinco), Moraxella (quatro), Serratia (três), Yersinia (dois), Klebsiella (um), Enterobacter (um) e Methylobacterium (um). Cinco bactérias não foram identificadas por meio do API 20E e API 20NE, que apresentaram resultados como "baixa discriminação, duvidoso ou inaceitável”. Pseudomonas foi o gênero mais isolado (43\%), sendo $\boldsymbol{P}$. fluorescens a espécie predominante (37,6\%). Esses resultados estão coerentes com os de outras pesquisas, que constataram predomínio desse gênero/espécie em leite cru (JAYARAO \& WANG, 1999; HOLM et al., 2004; ALATOSSAVA \& ALATOSSAVA, 2006; PINTO et al., 2006). A maior ocorrência de $\boldsymbol{P}$. fluorescens pode ser associada ao seu menor tempo de geração a temperaturas de refrigeração e também à sua habilidade em formar biofilme em superfícies de equipamentos e utensílios (JAYARAO \& WANG, 1999; HOLM et al., 2004).

Aeromonas hydrophila foi o patógeno predominante, com 20 isolados identificados (Tabela 1). Aeromonas hydrophila é um microrganismo de potencial patogênico emergente que causa infecções gastrintestinais (MERINO et al. 1995; DASKALOV, 2006). Esse microrganismo é freqüentemente encontrado na água (DASKALOV, 2006) e assim a água utilizada na limpeza de utensílios e equipamentos pode ser fonte importante de contaminação do leite e produtos lácteos.

Os gêneros/espécies patogênicos: Acinetobacter, Pantoea, Burkholderia cepacea (anteriormente Pseudomonas) e Klebsiella oxytoca também incluíram 10 ou mais isolados. A ocorrência dessa diversidade de espécies patogênicas é preocupante e estudos devem ser direcionados para a sua rastreabilidade por meio de sistemas acurados de identificação genotípica.

As bactérias gram-positivas foram identificadas à espécie quando o programa APILAB PLUS indicou identidade $>80,0 \%$ e a gênero quando indicou "boa identificação para gênero". Dos 58 isolados, apenas 22 foram identificados, sendo estes pertencentes aos gêneros Kurtia, Bacillus, Brevibacterium, Cellum/Microbacterium e Staphylococcus. A não identificação dos vários isolados pode ser atribuída ao número insuficiente de testes na galeria API e a limitações da base de dados do programa APILAB PLUS.

ALATOSSAVA\&ALATOSSAVA(2006), ao utilizarem o sistema API 20 NE para identificar 67 bactérias isoladas de leite cru, obtiveram para 29 isolados identificação “duvidosa”, "baixa discriminação" ou "inaceitável” e dois isolados não puderam ser identificados. Devido à limitação de identificação destes sistemas bioquímicos, deve-se considerar sua confirmação por caracterização genotípica.

Bactérias gram-negativas, particularmente Pseudomonas spp., são os principais responsáveis pela deterioração de leite refrigerado. Como esperado, a maioria das Pseudomonas apresentou atividade enzimática extracelular (Tabela 2). Todas $\boldsymbol{P}$. fluorescens foram lipolíticas a $4^{\circ} \mathrm{C}, 7^{\circ} \mathrm{C}, 10^{\circ} \mathrm{C}$ e $21^{\circ} \mathrm{C}$, sendo que a atividade proteolítica a estas temperaturas foi verificada, respectivamente, em 66\%, 74,5\%, 88,3\% e 95,7\% das estirpes. Do total de 108 Pseudomonas spp., $60,57 \%$ apresentaram as duas atividades de proteólise e lipólise. Os três isolados identificados como $\boldsymbol{P}$. putida foram proteolíticos e lipolíticos. Embora $\boldsymbol{P}$. fluorescens seja mais comumente associada à deterioração de leite, outras espécies como $\boldsymbol{P}$. putida, $\boldsymbol{P}$. fragi e $\boldsymbol{P}$. maltophila são também comuns (WIEDMANN et al., 2000). Os problemas ou defeitos atribuídos a Pseudomonas são rancidez, sabor amargo, sabor de fruta, geleificação em leite UHT, instabilidade térmica do leite, instabilidade do leite ao etanol, resultado falso-positivo na pesquisa por fraude de leite com soro por meio da dosagem do ácido siálico e redução no rendimento na fabricação de queijos (CHAMPAGNE et al.,1994; SØRHOUG \& STEPANIK, 1999; ARCURI et al., 2004).

Os gêneros Acinetobacter, Aeromonas e

Burkholderia, que incluem espécies patogênicas, também evidenciaram alto potencial de deterioração. (Tabela 2). Todas B. cepacea, a maioria dos Acinetobacter (94,9\%) e cerca de $50 \%$ das Aeromonas apresentaram atividade lipolítica em todas as temperaturas. Quanto à proteólise, esta foi verificada para A. caviae em todas as temperaturas estudadas, para $>50 \%$ das $\boldsymbol{A}$. hydrophila a $7^{\circ} \mathrm{C}, 10^{\circ} \mathrm{C}$ e $21^{\circ} \mathrm{C}$, mas para nenhuma $\boldsymbol{B}$. cepacea.

Bactérias psicrotróficas da família Enterobacteriaceae podem causar deterioração em leite (BOOR \& MURPHY, 2002). Dos 48 isolados, atribuídos a sete gêneros desta família (Pantoea, Klebsiella, Ewingella, Hafnia, Serratia, Yersinia e Enterobacter), nenhum apresentou atividade proteolítica a $4^{\circ} \mathrm{C}$, seis apresentaram a $7^{\circ} \mathrm{C}$ e dez apresentaram a $10^{\circ} \mathrm{C}$ e $21^{\circ} \mathrm{C}$. Atividade lipolítica foi verificada para um isolado a $4^{\circ} \mathrm{C}$, cinco a $7^{\circ} \mathrm{C}$ e nove a $10^{\circ} \mathrm{C}$ e $21^{\circ} \mathrm{C}$. A atividade enzimática desses microrganismos é espécie especifica e depende da temperatura de armazenamento (ALATOSSAVA \& 
Tabela 2 - Atividade enzimática de bactérias Gram-negativas e Gram-positivas nas temperaturas de $4^{\circ} \mathrm{C}, 7^{\circ} \mathrm{C}, 10^{\circ} \mathrm{C}$ e $21^{\circ} \mathrm{C}$.

\begin{tabular}{|c|c|c|c|c|c|c|c|c|}
\hline \multirow{3}{*}{ Gênero ou espécie (número de amostras) } & \multicolumn{8}{|c|}{-------------------No de estirpes positivas (produção de halo) ------------------- } \\
\hline & \multicolumn{4}{|c|}{-----------------Proteólise----------------- } & \multicolumn{4}{|c|}{------------------Lipólise------------------ } \\
\hline & $4^{\circ} \mathrm{C}$ & $7^{\circ} \mathrm{C}$ & $10^{\circ} \mathrm{C}$ & $21^{\circ} \mathrm{C}$ & $4^{\circ} \mathrm{C}$ & $7^{\circ} \mathrm{C}$ & $10^{\circ} \mathrm{C}$ & $21^{\circ} \mathrm{C}$ \\
\hline \multicolumn{9}{|l|}{ Bactérias Gram-negativas (250) } \\
\hline Pseudomonas fluorescens (94) & 62 & 70 & 83 & 90 & 94 & 94 & 94 & 94 \\
\hline Pseudomonas putida (3) & 3 & 3 & 3 & 3 & 3 & 3 & 3 & 3 \\
\hline Pseudomonas spp. (11) & 2 & 4 & 5 & 3 & 8 & 10 & 9 & 9 \\
\hline Acinetobacter spp. (39) & 0 & 4 & 4 & 1 & 37 & 37 & 29 & 37 \\
\hline Aeromonas hydrophila (20) & 1 & 10 & 14 & 14 & 10 & 11 & 10 & 11 \\
\hline Aeromonas caviae (1) & 1 & 1 & 1 & 1 & 1 & 1 & 1 & 1 \\
\hline Aeromonas sobria (1) & 0 & 0 & 1 & 1 & 0 & 0 & 1 & 1 \\
\hline Aeromonas spp. (5) & 0 & 1 & 5 & 5 & 2 & 3 & 3 & 3 \\
\hline Pantoea spp. (17) & 0 & 0 & 1 & 1 & 0 & 1 & 4 & 4 \\
\hline Burkhol. Cepacia (12) & 0 & 0 & 0 & 0 & 12 & 12 & 9 & 12 \\
\hline Klebsiella oxytoca (10) & 0 & 3 & 3 & 3 & 0 & 1 & 1 & 1 \\
\hline Klebsiella sp.(1) & 0 & 1 & 1 & 1 & 1 & 1 & 1 & 1 \\
\hline Ewingella americana (7) & 0 & 0 & 1 & 1 & 0 & 0 & 0 & 0 \\
\hline Hafnia Alvei (7) & 0 & 0 & 0 & 0 & 0 & 0 & 0 & 0 \\
\hline Moraxella spp. (4) & 0 & 0 & 0 & 0 & 4 & 4 & 2 & 4 \\
\hline Chryseomonas luteola (3) & 2 & 2 & 1 & 2 & 2 & 2 & 2 & 1 \\
\hline Serratia spp. (3) & 0 & 0 & 2 & 1 & 0 & 0 & 2 & 1 \\
\hline Yersinia spp. (2) & 0 & 2 & 2 & 2 & 0 & 2 & 2 & 2 \\
\hline Enterobacter sp. (1) & 0 & 0 & 0 & 0 & 0 & 0 & 0 & 0 \\
\hline Methylobacterium sp. (1) & 0 & 0 & 0 & 0 & 1 & 1 & 1 & 1 \\
\hline Alcaligenes faecalis (1) & 0 & 0 & 0 & 0 & 1 & 1 & 1 & 1 \\
\hline Pasteurella sp. (1) & 0 & 0 & 0 & 1 & 1 & 1 & 1 & 1 \\
\hline Sphingomonas Paucimobilis (1) & 0 & 0 & 0 & 0 & 0 & 0 & 1 & 1 \\
\hline Não identificado (5) & 0 & 0 & 1 & 2 & 4 & 5 & 5 & 5 \\
\hline \multicolumn{9}{|l|}{ Bactérias Gram-positivas (58) } \\
\hline Kurtia spp. (7) & 0 & 0 & 0 & 0 & 0 & 0 & 0 & 0 \\
\hline Bacillus coagulans (1) & 0 & 1 & 1 & 1 & 0 & 0 & 0 & 0 \\
\hline Bacillus lentus (2) & 0 & 2 & 2 & 2 & 0 & 0 & 0 & 0 \\
\hline Bacillus sp (1) & 0 & 0 & 1 & 0 & 0 & 0 & 1 & 1 \\
\hline Brevibacterium (1) & 0 & 1 & 1 & 1 & 0 & 0 & 0 & 0 \\
\hline Cellum/Microbacterium (6) & 0 & 6 & 6 & 6 & 0 & 0 & 0 & 0 \\
\hline Staphylococcus spp. (3) & 0 & 0 & 0 & 0 & 0 & 0 & 0 & 0 \\
\hline Não identificados (37) & 0 & 19 & 29 & 27 & 5 & 6 & 13 & 21 \\
\hline Total (308) & 71 & 130 & 168 & 168 & 186 & 196 & 196 & 216 \\
\hline
\end{tabular}

ALATOSSAVA, 2006), como evidenciado por esses dados.

Dos 58 isolados gram-positivos, nenhum apresentou atividade proteolítica a $4^{\circ} \mathrm{C}$, mas cinco apresentaram atividade lipolítica a esta temperatura (Tabela 2). Já $50 \%$ desses microrganismos expressaram atividade proteolítica a $7^{\circ} \mathrm{C}$ e a maioria expressou a $10^{\circ} \mathrm{C} \mathrm{e} 21^{\circ} \mathrm{C}$, indicando que temperaturas abaixo de $4^{\circ} \mathrm{C}$ controlam bem a atividade proteolítica destes microrganismos. Em geral, as bactérias gram-positivas foram encontradas com menor freqüência e predominaram dentre os isolados de cinco amostras de leite.

\section{CONCLUSÃO}

Os resultados obtidos demonstram o nível de contaminação de leite cru com bactérias psicrotróficas e que a mistura de matéria-prima de diversos produtores em tanques coletivos pode aumentar os riscos de contaminação. Além disso, os resultados mostram que o grupo psicrotrófico inclui uma diversidade de gêneros bacterianos, com predominância do gênero Pseudomonas, e que em sua maioria produz proteases e/ou lípases a temperaturas de refrigeração. Esses resultados evidenciam a necessidade de serem realizados, nas propriedades 
rurais, treinamento, implementação e monitoramento contínuo de boas práticas para prevenir contaminação e crescimento microbiano no leite.

\section{AGRADECIMENTOS}

Os autores agradecem à Fundação de Amparo à Pesquisa do Estado de Minas Gerais (FAPEMIG) (projeto CAG 180) e ao Conselho Nacional de Desenvolvimento Científico e Tecnológico (CNPq) (projeto 472436), pelo apoio financeiro. Priscilla Diniz Lima da Silva foi bolsista da CAPES.

\section{REFERÊNCIAS}

ALATOSSAVA, P.M.; ALATOSSAVA, T. Phenotypic characterization of raw milk-associated psychrotrophic bacteria. Microbiological Research, v.161, p.334-346 2006 .

ARCURI, E.F. et al. Efeito do crescimento de Pseudomonas sp. proteolítica na estabilidade do leite ao etanol. In: CONGRESSO NACIONAL DE LATICÍNIOS, 21., Juiz de Fora, MG. Revista do ILCT v.59, n.339, p.140-144, 2004.

BARROW, G.I.; FELTHAM, R.K.A. (ed.). Cowan and Steel's manual for the identification of medical bacteria. 3.ed. Cambridge: Cambridge University, 1993. 331p.

BOOR, K.J.; MURPHY, S.C. Microbiology of market milk. In: ROBINSON, R.K. Dairy microbiology handbook. 3.ed. New York: John Wiley and Sons, 2002. p.91-122.

BRITO, M.A.V.P. et al. Identificação de contaminantes bacterianos no leite cru de tanques de refrigeração. In: CONGRESSO NACIONAL DE LATICÍNIOS, 19., 2002, Juiz de Fora, MG. Revista do ILCT, v.57, p.83-88, 2002.

BRUM, J.V.F. et al. Pesquisa de microrganismos psicrotróficos em leite cru produzido nos estados do Paraná e Santa Catarina. In: CONGRESSO NACIONAL DE LATICÍNIOS, 21., 2004, Juiz de Fora, MG. Revista do ILCT, v.59, p.150-154, n. 339, 2004.

CHAMPAGNE, C.P. et al. Psychrotrophs in dairy products : their effects and their control. Critical Review in Food Science and Nutrition, v.34, p.1-30, 1994.
CHEN, L. et al. Detection and impact of protease and lipase activities in milk and milk powders. International Dairy Journal, v.13, p.255-275, 2003.

DASKALOV, H. The importance of Aeromonas hydrophila in food safety. Food Control, v.17, p.474-483, 2006.

FRANK, J.F. et al. Tests for groups of microrganisms. In: MARSHALL, R.T. (Ed.). Standard methods for the examination of dairy products. 16.ed. Washington: American Public Health Association, 1992. p.271-286.

HOLM, L.J. et al. Predominant microflora of downgraded danish bulk tank milk. Journal of Dairy Science, v.87, p.1151-1157, 2004.

JAYARAO, B.M.; WANG, L. A study on the prevalence of Gram-negative bacteria in bulk tank milk. Journal of Dairy Science, v.88, p.2620-2624, 1999

MERINO, S. et al. Emerging pathogens: Aeromonas spp. Food microbiology, v.28, p.157-168, 1995

PINTO, C.L.O. et al. Qualidade microbiológica de leite cru refrigerado e isolamento de bactérias psicrotróficas proteolíticas. Ciência e Tecnologia de Alimentos, v.26, n.3, p.645-651, 2006.

SHAH, N.P. Psychrotrophs in milk: a review. Milchwissenschaft, v.49, p.432-437, 1994.

SØRHOUG, T.; STEPANIAK, L. Psychrotrophs and their enzymes in milk and dairy products: quality aspects. Trends in Food Science and Technology, Cambridge, v.8, p.35-41, 1997.

SOUZA, M.R. et al. Avaliação da qualidade do leite resfriado, estocado em propriedades rurais por 48 horas e recebido por uma indústria de laticínios. In: CONGRESSO NACIONAL DE LATICÍNIOS, 16., 1999, Juiz de Fora, MG. Revista do ILCT v.54, p.238-241, 1999.

WIEDMANN, M. et al. Molecular and phenotypic characterization of Pseudomonas spp. isolated from milk. Applied and Environmental Microbiology, v.66, p.20852095, 2000 . 